\title{
Influences impacting the attitudes of parents towards the HPV vaccine
}

\begin{abstract}
Background: HPV virus is responsible for $90 \%$ of invasive cervical cancers. The overall aim of this study was to identify the influences behind attitudes currently held by parents and the factors causing the decrease in uptake.
\end{abstract}

Materials and methods: An anonymous questionnaire was designed and circulated to 158 individuals. Questionnaire included both quantitative and qualitative questions. Outcome measures were HPV knowledge, information sources and parental attitudes.

Results: Response rate was $26 \%$. A total of $68.3 \%$ of parents had good knowledge of the HPV virus/vaccine. A strong trend towards significance in this study suggested that participants with lower knowledge were more likely to consent. Concerns about the vaccine safety were the main barrier to vaccination. Increased knowledge would indicate that the parents were more likely to perform in-depth research and discover the well-publicised safety concerns.

Conclusion: Further efforts must be made to counteract the negative media available to parents to ensure they are fully informed in their decision making. Further research on the parents' knowledge of vaccine safety could be done to address this.

Keywords: human papilloma virus vaccine, HPV vaccine, vaccinations, vaccine hesitancy
Volume II Issue I - 2020

\author{
Kate Lawless, Patrick Murphy, Laure \\ Marignol, Claire Poole \\ Discipline of Radiation Therapy, School of Medicine, Trinity \\ College Dublin, University of Dublin, Ireland
}

Correspondence: Claire Poole, Trinity College Dublin,

Applied Radiation Therapy Trinity Research Group, Discipline of

Radiation Therapy, School of Medicine, Dublin, Ireland, Tel +353-

I-896-2973 Fax +353-I-896-3246, Email poolc@tcd.ie

Received: December 17, 2019 | Published: January 21, 2020

\section{Introduction}

Cervical Cancer is the 4 th most common cancer worldwide, representing $6.6 \%$ of all female cancers in $2018 .{ }^{1}$ The top risk factor for developing invasive cervical cancer is Infection with high-risk Human Papilloma Virus (HPV). There are approximately $13 \mathrm{HPV}$ types classified as high. ${ }^{2,3} \mathrm{HPV}$ also has associations with cancer types such as anal, vulvar, penile and some cancers of the head and neck region. $^{4-6}$

Cervarix was the first vaccine introduced worldwide. It was a bivalent vaccine, which targeted HPV types 16 and 18. In more recent years, a quadrivalent vaccine was introduced clinically with the brand name, Gardasil. This vaccine targets types 6,11, 16 and 18. Types 6 and 11 have associations with the development of genital warts. ${ }^{7,8}$ In 2016, the Lancet journal published an article which stated that elimination of HPV types $6,11,16$ and 18 would be possible if $80 \%$ target coverage in both boys and girls is reached and if high vaccine efficacy is maintained over time. ${ }^{9}$ A ten-year follow-up study from Finland found that ten-year statistically significant vaccine efficacy of $66 \%$ for HPV 16/18 had been achieved. ${ }^{10}$ In 2014 , a nonavalent vaccine was licensed and approved by the Food and Drug Administration (FDA). ${ }^{11}$ This nonavalent (9vHPV) vaccine was approved for use in the European Union in 2015. This vaccine will protect against 9 subtypes of HPV; 6, 11, 16, 18, 31, 33, 42, 52 and 58. ${ }^{12}$ This vaccine was produced to ensure a higher protection against HPV-related malignancies, thus creating a more effective vaccination programme.

Research conducted prior to the introduction of the vaccine indicated that there would be a favourable uptake upon its introduction. ${ }^{13}$ The review looked at the reasons why parents were willing/not willing to vaccinate their children. Their results indicated that belief in the effectiveness of the vaccine led to the increased likelihood that they would vaccinate their daughters. The barriers to vaccination included concern of increased adolescent sexual activity and fears regarding the safety of the vaccine. ${ }^{8}$

Bruni et al. ${ }^{14}$ in their global review of HPV uptake indicated that Europe and North America had less than 50\% uptake with the primary target group. Uptake of the vaccine determines the success of the prophylactic vaccination programmes; $80 \%$ coverage is needed to reduce the cancer burden associated with HPV. ${ }^{15}$ According to WHO, the vaccine is available in 74 countries, however there is less than $50 \%$ coverage; $31 \%$ in Europe, $35.6 \%$ in North America, $1.1-1.2 \%$ in Africa and Asia. ${ }^{16}$ In Ireland, the HPV vaccine uptake dropped from $84.9 \%$ in $2013 / 2014$ to $51 \%$ in $2016 / 2017^{17}$ with worries regarding the safety of the HPV vaccine as the reason underlying drop in uptake. The safety of the vaccine has been explored in the literature. ${ }^{18,19} \mathrm{~A}$ meta-analysis found an insignificant difference between serious adverse events reported between vaccinated and control groups. ${ }^{18}$ The percentage of vaccine-related serious adverse events ranged from $0-0.1 \%{ }^{18}$ In addition to structural and health funding issues, vaccine hesitancy of parents appears to have a significant influence on HPV vaccine uptake. ${ }^{16}$

The focus of the study was to gain a deeper understanding of what is currently influencing parents' decision to vaccinate their child against the HPV vaccine. 


\section{Objectives}

a. To establish parent's current knowledge of the HPV virus and the HPV vaccine,

b. Ascertain the current attitudes of parents towards the HPV vaccine and of vaccines in general and

c. Determine what is influencing the attitudes currently held by parents towards vaccines/HPV vaccine.

\section{Materials and methods}

Ethical approval for this study was sought and gained from the relevant university Research Ethics Committee. An original questionnaire was designed, piloted and amended by public as appropriate. Questionnaire contained six sections; Section 1: Demographics of the participant population. Section 2: Knowledge of the HPV virus and vaccine. Section 3: Sources of information about the HPV vaccine and level of research conducted into the vaccine. Section 4: Childhood vaccinations and vaccines in general. Section 5: Attitudes towards vaccines, HPV vaccine and HPV virus (General Vaccines, HPV Vaccine, Sexual Behaviour and the HPV Vaccine and HPV Vaccine Safety). The section concluded with a qualitative question asking for further comments on any of the above statements. Section 6: Attitudes towards the HPV Vaccine. Participants were asked to state if they were intending to give consent for the vaccine and state any opinions they have on the HPV Vaccine/Virus.

\section{Target population}

Parents/guardians of first-year female secondary school students. Exclusion criteria included anyone under the age of 18, parents/ guardians whose daughters were not in first year or who had already received the vaccine.

\section{Data collection}

An email including a participant recruitment letter was sent to six secondary schools. Two schools replied confirming interest in the study. This created a participant population of 158 parents/guardians. Students' were asked to deliver the questionnaires to their parents/ guardians to complete if they wished to take part. The participants were given 21 days to return the questionnaires to their daughter's school, in a sealed envelope which had been provided for them. The final participant population was 41 .

\section{Data analysis}

Statistical Package for the Social Sciences (SPSS) was used to analyse the data. Chi-Square tests were used to establish relationships between different variables and likelihood to vaccinate. If the number of responses were less than 5, Fischer's Exact Tests were run instead. Some variables were recoded to allow for Chi square tests and fisher exact tests to be run. A spearman correlation test was run to determine if a relationship existed between age and knowledge of HPV. When analyzing the attitudes of parents, the mean, mode, median and standard deviation values of the Likert scales were analyzed. A thematic analysis was performed on the qualitative aspects of the questionnaire. The thematic analysis was guided by methodology from Braun et al. ${ }^{20}$

\section{Results}

The final participant population was 41 with a response rate of $25.9 \%(41 / 158)$. There was no exclusion of questionnaires. The proposed vaccine uptake from this study was $63.45 \%(n=26)$. A significant result was one indicated by a p-value $<0.05$.

\section{Demographic factors}

A list of the demographic factors and their frequencies are seen in Table 1. A Chi-square test was utilised to test the relationship between age, religion, ethnicity and education with likelihood to vaccinate their child. The test revealed a trend towards significance between age and likelihood to vaccinate $(\mathrm{p}=0.297)$. The trend suggests that increasing age, increases a person's likelihood to vaccinate. There was no significant relationship identified between parents' religion/ ethnicity/education and likelihood to vaccinate $(\mathrm{p}=0.627,0.923$, 0.440 respectively).

Table I Qualitative analysis:Thematic analysis

\begin{tabular}{lll}
\hline Identified themes & $\mathbf{n}=$ & $\% *$ \\
\hline "Believes in importance of vaccines" & 7 & 23.3 \\
"Worries about side-effects controversy" & 4 & 13.3 \\
"Wishes to prevent cervical cancer" & 16 & 53.3 \\
"Worries about safety of vaccine" & 6 & 20 \\
"Worries regarding increased sexual behaviour" & I & 3.33 \\
"Lack of available information for parents" & 3 & 10 \\
\hline
\end{tabular}

*\% of participants who answered the qualitative section $(n=30)$

\section{HPV knowledge}

A strong trend towards significance was identified between knowledge of HPV and likelihood to vaccinate using a chi-square test $(p=0.067)$. The knowledge scores were marked out of 11 . The participants scores ranged from 2-11, these results are displayed in Figure 1. A Spearman's correlation test proved there was no significant linear relationship between age and knowledge score $(\mathrm{rs}(41)=0.075$, $\mathrm{p}=0.640$ ).

\section{HPV Knowledge Scores}

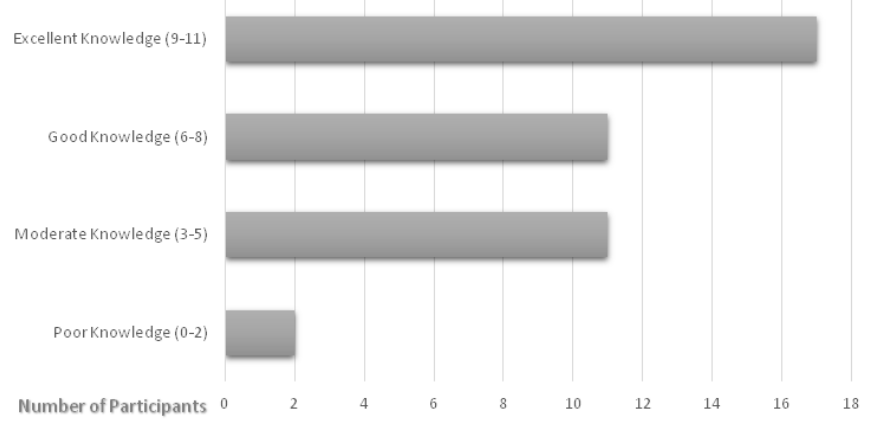

Figure I HPV knowledge scores.

\section{Information sources}

Of the participant population, $92.7 \%(n=38)$ had heard of the HPV vaccine before completing the questionnaire. Range of sources of information is displayed in Figure 2. A significant relationship was identified between using 'friends' as a source of information and likelihood to vaccinate $(\mathrm{p}=0.045)$. 


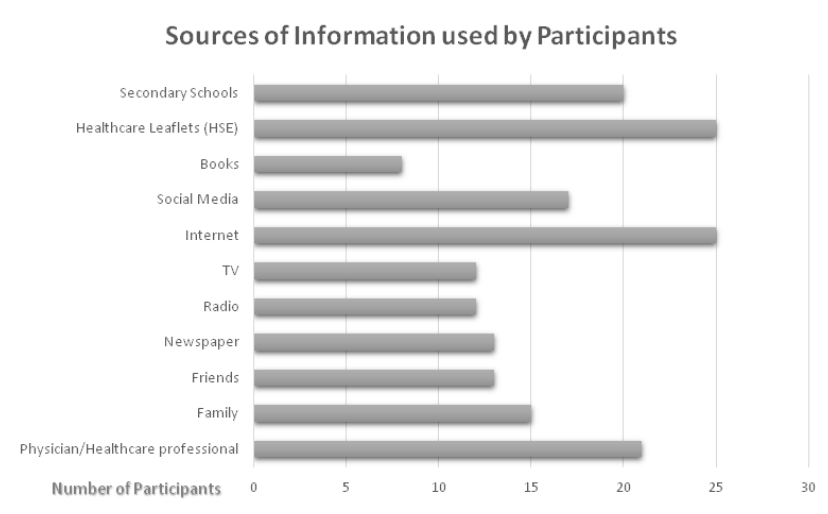

Figure 2 Sources of information used by participants.

\section{Vaccine research}

The participants were asked if they intended to conduct research into the vaccine, $71.1 \%(\mathrm{n}=27)$ answered 'Yes'. A Chi-square indicated no significant relationship between intention to research and likelihood to vaccinate $(\mathrm{p}=0.326)$. A weak trend towards significance was discovered between length of time spent researching and likelihood to vaccinate $(\mathrm{p}=0.253)$.

\section{Parent's attitudes}

In Section 5.1 attitudes towards General Vaccines were measured. The mean values ranged from 3.41-4.12. There was an overall positive attitude towards general vaccines. In Section 5.2, attitudes towards the HPV vaccine were measured. The mean values ranged from 3.49-3.71. This shows an overall neutral attitude towards the HPV vaccine. In Section 5.3, attitudes towards Sexual Behaviour and the HPV Vaccine were measured. A median value of 2.00 (Disagree) was given to $4 / 5$ of the statements. The mean values ranged from 1.95 3.83. This indicates that the parents disagreed with the statements regarding the HPV vaccine and increased sexual behaviour. In Section 5.4, the parents' attitude towards the safety of the HPV vaccine was measured. The mean values ranged from 3.32-3.95. This indicated that the parents were worried overall about the safety of the vaccine and the side-effects that have been reported in the media.

\section{Thematic analysis}

For the qualitative questions a thematic analysis was performed. Only 30 of the participants answered the voluntary qualitative sections of the questionnaire. The most frequently occurring theme was the parents' wish to protect their child from cervical cancer and trusting the HPV vaccine to achieve this $(\mathrm{n}=16)$. The second most frequently occurring them was the parents' belief in the importance of vaccines $(n=7)$.

\section{Discussion}

In this study, the weak trend towards significance in relation to age $(\mathrm{p}=0.297)$, gave an indication that participants in the youngest age group (age 26-35 years), were more likely not to give consent when compared to the older age groups. Reiter et al. also found a weak association between parents' age and vaccine acceptability, ${ }^{21}$ which is reflective of the results of this study. However, they grouped parent's age as $<40$ or $>40$ years of age, unlike our study. They did not report the percentage of the age groups who consented to the vaccine, so it is not possible to compare the trend in age with our results.
Constantine et al. also looked at parents age but found no significant effects between age and acceptability. ${ }^{22}$ The trend found in Brabin et al. ${ }^{23}$ showed that with increasing age, there was less acceptability of the vaccine.

Our results showed no significant relationship between religion and likelihood to vaccinate. This contradicts evidence from the literature. Constantine et al. found a significant relationship between religion and acceptability. ${ }^{22}$ The Catholic and other Christian groups demonstrated significance values of $(p=0.001$ and $p=0.006$ respectively). This is in stark contrast to the results of our study. Our results contradict with evidence presented in a study by Brabin et al. ${ }^{23}$ published in 2006 . This study found a trend towards significance of religion vs. acceptability, when ordinal regression was performed $(\mathrm{p}=0.160) .{ }^{22}$ This study had a much larger sample size of 317 participants, which may also be the reason that a trend towards significance was identified.

Brabin et al. ${ }^{23}$ also reported that there was no significance between ethnicity and likelihood to consent $(\mathrm{p}=0.730)$. These results for ethnicity were reflected in our study. Brabin et al. ${ }^{23}$ had a majority of white participants $(66.3 \%)$, which is similar to our study. Similar results and similar ethnicity profiles were found in our trial and the trial by Brabin et al. ${ }^{23}$ Constantine et al. ${ }^{22}$ also had a majority White population, but with an almost equal Hispanic ethnicity group. This study found significance between ethnicity groups and vaccine acceptability, unlike our study and the study by Brabin et al. ${ }^{23}$

It was assumed that better knowledge of HPV and the HPV vaccine, would increase the likelihood of consenting to the vaccine. The strong trend towards significance found in our study suggested that participants with a lower knowledge were more likely to consent. This conflicts with our assumptions prior to the commencement of the study. Dempsey et al. ${ }^{24}$ put forward the same hypothesis. They assumed that increased knowledge scores would lead to increased acceptability of the vaccine. Dempsey et al. ${ }^{24}$ had two groups, one was given an HPV information sheet and the other was not. Their results showed no significant increase in acceptability in the test group in comparison with the control group. A study from 2015 concluded that those with higher levels of knowledge were not more likely to consent to the vaccine, ${ }^{25}$ which is consistent with the results from Dempsey et al.,${ }^{24}$ Unger et al. ${ }^{26}$ found that awareness of HPV was high but there were gaps in the HPV knowledge. A possible explanation for our results could be that increased knowledge of HPV may indicate an increase in research conducted into the vaccine. This might lead to an increased exposure to worrying side effects reported in the media. The gaps in knowledge mentioned by Unger et al. may be another explanation for our results, if these gaps were regarding the safety of the vaccine (26). Further testing on the knowledge of vaccine safety could be done to confirm this.

Literature demonstrated that the way parents source information may be an important factor in their decision-making process. Research shows that the use of a physician as an information source, increased the likelihood of consenting to the vaccine. ${ }^{13}$ Rosenthal et al. ${ }^{27}$ found the use of a physician increased the likelihood of vaccination sevenfold. Despite this consensus among the literature, when tested in our study, no significant relationship was determined between the use of a physician and likelihood to vaccinate. Brewer et al. ${ }^{13}$ suggest that it is the recommendation given by the physician which will influence the parent's decision. Our study did not test the recommendation given to the participants by the physician. Therefore, our results can only be based on the use of a physician. 
Hughes et al. ${ }^{28}$ proposed that parents who had heard of the vaccine from a family member/friend were more likely to consent. This contradicts our results. Our study found a significant relationship between consulting friends and likelihood to vaccinate. The trend suggested that participants that did not consult their friends were more likely to consent. The same trend was found when family members were consulted. The Hughes et al. ${ }^{28}$ study was published in 2009, prior to the side effects media controversy. It is possible that in more recent times, parents are less likely to consult family/friends due to conflicting opinions of the general public with regards to the vaccine. Allen et al. ${ }^{29}$ assessed the social influence of family and friends on decision-making and found a weak association between them. It stated that more significant factors included HPV Knowledge, awareness and attitudes towards the vaccine.

Our study found that $97.6 \%(n=40)$ of the participants consented to their daughter receiving her childhood vaccinations. These results could be explained by the long-time established acceptability of childhood vaccines such as the MMR vaccine (Measles, Mumps and Rubella). Although currently well established and generally trusted, the MMR vaccine underwent a period of distrust in the media and among the public. The unsubstantiated claims made by Andrew Wakefield in relation to the MMR vaccine's links with Autism, contributed to declines in MMR vaccine coverage. ${ }^{30,31}$ These claims caused a drop-in uptake levels to less than $80 \%$ in the UK in the years 2003/04. ${ }^{32}$ Although lower than WHO recommendations, the MMR vaccine has recovered from the Wakefield controversy in recent years in the UK and Ireland..$^{32,33}$ Larson et al..$^{34}$ discusses the many vaccines which have undergone periods of decline during their history for example MMR, Hepatitis-B, Tetanus and others. He states that trust in the MMR vaccine would not have recovered without first recovering the trust lost in healthcare professionals and the government. This pattern of recovery could be used to restore trust in the HPV vaccine.

Overall, the parents had a positive attitude towards general vaccines. From the statements asked regarding sexual behaviour and HPV vaccines, the parents' responses suggested that increased sexual behaviour was not a concern for them. This conflicts with evidence in the literature which identifies the worries regarding increased sexual behaviour as a barrier towards the vaccine..$^{22,35,36}$

In our study, a major barrier that was highlighted was the belief that short-term and long-term side effects of the vaccine could impact on their daughters' health negatively. In particular, the ones which have been highlighted recently in the media. The two main conditions include Complex Regional Pain Syndrome (CRPS) and Postural Orthostatic Tachycardia Syndrome (POTS). A review published by the European Medicines Agency in 2015, concluded there was no causal relationship between the vaccine and these two conditions. ${ }^{37} \mathrm{~A}$ phase III trial also found that the numbers of serious adverse events related to the vaccine were $0.1 \%$ in both the vaccine and the control group. ${ }^{38}$

The limitations of this study include the small sample size of 41 . It is difficult to achieve any statistically significant results with a sample size of less than 50. One further limitation could include bias. As both schools sampled are located close to each other, it is possible there may be bias and the results gathered may not be representative of the national population. However, schools with different socio-economic backgrounds were selected to reduce this bias as much as possible.

\section{Conclusion}

This study concludes with the statement that most parents included in the study intend to vaccinate their daughter (63.4\%). The biggest barrier to vaccination identified is fears regarding the safety of the vaccine and worries related to associated side-effects. These fears and worries were also present among the group who gave consent for the vaccine. Further efforts must be made to alleviate these fears and reassure parents that the vaccine is safe. Parents need to be given easy access to more information on vaccine safety, in an effort to increase the uptake of the HPV vaccine. Assumptions that more knowledge of HPV would lead to acceptability was not established in this study, instead it was suggested that those with lower knowledge were more likely to consent. Increased knowledge would indicate that the parents were more likely to do in-depth research and discover the well-publicised safety concerns. Further efforts must be made to counteract the negative media available to parents to ensure they are fully informed in their decision making.

\section{Acknowledgments}

None.

\section{Conflicts of interest}

The authors declare there is no conflict of interest.

\section{Funding}

None.

\section{References}

1. Cervical Cancer. World Health Organization. 2019.

2. Kabekkodu S, Bhat S, Pandey D, et al. Prevalence of human papillomavirus types and phylogenetic analysis of HPV-16 L1 variants from Southern India. Asian Pac J Cancer Prev. 2015;16(5):2073-2080.

3. Argyri E, Tsimplaki E, Papatheodorou D, et al. Recent Trends in HPV Infection and Type Distribution in Greece. Anticancer Res. 2018;38(5):3079-3084.

4. Roberts J, Siekas L, Kaz A. Anal intraepithelial neoplasia: A review of diagnosis and management. World J Gastrointest Oncol. 2017;9(2):5061.

5. Kim SM. Human papilloma virus in oral cancer. J Korean Assoc Oral Maxillofac Surg. 2016;42(6):327-336.

6. Marur S, D'Souza G, Westra W, et al. HPV-associated head and neck cancer: a virus-related cancer epidemic. Lancet Oncol. 2010;11(8):781789

7. Kessels S, Marshall H, Watson M, et al. Factors associated with HPV vaccine uptake in teenage girls: A systematic review. Vaccine. 2012;30(24):3546-3556

8. Joura E, Pils S. Vaccines against human papillomavirus infections: protection against cancer, genital warts or both? Clin Microbiol Infect. 2016;22:S125-S127.

9. Brisson M, Bénard É, Drolet M, et al. Population-level impact, herd immunity, and elimination after human papillomavirus vaccination: a systematic review and meta-analysis of predictions from transmissiondynamic models. Lancet Public Health. 2016;1(1):e8-e17.

10. Lehtinen M, Lagheden C, Luostarinen $\mathrm{T}$, et al. Ten-year follow-up of human papillomavirus vaccine efficacy against the most stringent cervical neoplasia end-point-registry-based follow-up of three cohorts from randomized trials. BMJ Open. 2017;7(8):e015867.

11. Lopalco P. Spotlight on the 9-valent HPV vaccine. Drug Des Devel Ther. 2016;11:35-44.

12. Riethmuller D, Jacquard A, Lacau St Guily J, et al. Potential impact of a nonavalent HPV vaccine on the occurrence of HPV-related diseases in France. BMC Public Health. 2015;15(1):1-7. 
13. Brewer N, Fazekas K. Predictors of HPV vaccine acceptability: A theory-informed, systematic review. Prev Med. 2007;45(2-3):107-114.

14. Bruni L, Diaz M, Barrionuevo-Rosas, et al. Global estimates of human papillomavirus vaccination coverage by region and income level: a pooled analysis. Lancet Glob Health. 2016;4(7):e453-e463.

15. Loke AY, Kwan ML, Wong YT, et al. The uptake of human papillomavirus vaccination and its associated factors among adolescents: a systematic review. J Prim Care Community Health. 2017;8(4):349-362.

16. Newman AP, Logie HC, Lacombe-Duncan A, et al. Parents' uptake of human papillomavirus vaccines for their children: a systematic review and meta- analysis of observational studies. BMJ Open. 2018;8(4):1-15.

17. HPV Immunisation Uptake Statistics. Health Protection Surveillance Centre, 2018.

18. Lu B, Kumar A, Castellsagué X, et al. Efficacy and Safety of Prophylactic Vaccines against Cervical HPV Infection and Diseases among Women: A Systematic Review \& Meta-Analysis. BMC Infect Dis. 2011;11(1):1-16.

19. Pomfret T, Gagnon Jr J, Gilchrist A. Quadrivalent human papillomavirus (HPV) vaccine: a review of safety, efficacy, and pharmacoeconomics. $J$ Clin Pharm Ther. 2011;36(1):1-9.

20. Braun V, Clarke V. Using thematic analysis in psychology. Qualitative Research in Psychology. 2006;3(2):77-101.

21. Reiter P, Brewer N, Gottlieb S, et al. Parents' health beliefs and HPV vaccination of their adolescent daughters. Soc Sci Med. 2009;69(3):475480 .

22. Constantine N, Jerman P. Acceptance of Human Papillomavirus Vaccination Among Californian Parents of Daughters: A Representative Statewide Analysis. J Adolesc Health. 2007;40(2):108-115.

23. Brabin L, Roberts S, Farzaneh F, et al. Future acceptance of adolescen human papillomavirus vaccination: A survey of parental attitudes. Vaccine. 2006;24(16):3087-3094.

24. Dempsey A. Factors that are associated with parental acceptance of human papillomavirus vaccines: a randomized intervention study of Written Information About HPV. Pediatrics. 2006;117(5):1486-1493.

25. Fishman J, Taylor L, Kooker P, et al. Parent and Adolescent Knowledge of HPV and Subsequent Vaccination. Pediatrics. 2014;134(4):e1049e1056.
26. Unger Z, Maitra A, Kohn J, et al. Knowledge of HPV and HPV Vaccine among Women Ages 19 to 26. Women's Health Issues. 2015;25(5):458462

27. Rosenthal S, Weiss T, Zimet G, et al. Predictors of HPV vaccine uptake among women aged 19-26: Importance of a physician's recommendation. Vaccine. 2011;29(5):890-895.

28. Hughes J, Cates J, Liddon N, et al. Disparities in How Parents Are Learning about the Human Papillomavirus Vaccine. Cancer Epidemio Biomarkers Prev. 2009;18(2):363-372.

29. Allen J, Othus M, Shelton R, et al. Parental Decision Making about the HPV Vaccine. Cancer Epidemiol Biomarkers Prev. 2010;19(9):21872198.

30. Burgess D, Burgess M, Leask J. The MMR vaccination and autism controversy in United Kingdom 1998-2005: Inevitable community outrage or a failure of risk communication? Vaccine. 2006;24(18):39213928.

31. DeStefano F. Vaccines and Autism: Evidence Does Not Support a Causal Association. Clin Pharmacol Ther. 2007;82(6):756-759.

32. Smith R. MMR uptake rates finally recovered from Wakefield scandal figures show. The Telegraph. 2017

33. Culliton G. MMR rates stand at 93\%. Irish Medical Times. 2016.

34. Larson H, Cooper L, Eskola J, et al. Addressing the vaccine confidence gap. Lancet. 2011;378(9790):526-535.

35. Mather T, McCaffery K, Juraskova I. Does HPV vaccination affect women's attitudes to cervical cancer screening and safe sexual behaviour? Vaccine. 2012;30(21):3196-3201.

36. Madhivanan P, Li T, Srinivas V, et al. Human papillomavirus vaccine acceptability among parents of adolescent girls: Obstacles and challenges in Mysore, India. Prev Med. 2014;64:69-74.

37. HPV Vaccines: EMA confirms evidence does not support that they cause CRPS or POTS. European Medicines Agency. United Kingdom. European Medicines Agency, 2016.

38. Paavonen J, Jenkins D, Bosch F, et al. Efficacy of a prophylactic adjuvanted bivalent L1 virus-like-particle vaccine against infection with human papillomavirus types 16 and 18 in young women: an interim analysis of a phase III double-blind, randomised controlled trial. Lancet. 2007;369(9580):2161-2170 